\title{
AN EMBEDDABLE SELF-REFLECTIVE SELF-ADAPTIVE SOFTWARE MODEL FOR RESOURCE MANAGEMENT
}

\author{
Adenowo A. A.", Yussuff A. I. O., Oluyemi O. T., Momoh K. \\ Dept. of Electronic \& Computer Engineering, Faculty of Engineering, Lagos State University, Lagos, Nigeria. \\ "Corresponding Author email: adetokyom@yahoo.com
}

Received: $12^{\text {th }}$ July 2018

Accepted: $26^{\text {th }}$ April 2019

Published: $17^{\text {th }}$ September 2019

https://doi.org/10.47545/etrj.2019.4.2.053

\begin{abstract}
With the growing complexity of today's world, there is rise in the design of smart and autonomous technologies. These technologies (or systems) are usually software-intensive and should have ability to self-manage, optimize resources, eliminate or reduce human intervention and still achieve quality and optimal output(s). Thus, technologies of this nature should self-reflect and self-adapt to internal and external factors to achieve its desired goal. Self-reflective, selfadaptive concepts are (or can be) used in a number of different areas including: robotics systems, autonomous computing, space systems, manufacturing, recommender systems, and e-commerce (for transactions monitoring), among others. These technologies basically have software component, which are usually underpinned by model(s). The main goal of this research, therefore, is to develop an embeddable software model (with self-reflective, selfadaptive properties) that shows real-time resource management. In developing our model, we particularly took into cognizance MAPE-K template for identification of self-* properties and performance requirements. We applied a Case Study within a particular domain (the education sector) to demonstrate the usability of this software model in managing human resource (a primary resource). The software model is able to reflect and modify its behavior at run-time in response to internal and external factors. Evaluation results show that the software model successfully self-manage the deployment of human resources; it was able to determine over/under-staffing, and redistribute (and/or identify recruitment needs for) human resource within the domain under consideration.
\end{abstract}

Keywords: - Resource Management, Self-Adaptive, Self-Reflective, Software Model.

\section{INTRODUCTION}

As new technologies emerge for managing the ever-increasing complexity of modern day activities and the growing inability of human to predict the cause-effects of interactions of deployed resources, it becomes obvious the need for software-intensive mechanisms that automate and simplify adaptation, management of performance and efficient resource allocations after deployment. This necessitate the design and continuous improvement of software systems with self-star (self-*) computing properties (self-reflective, self-adaptive, self-configuring, self-optimization, etc.). However, the design of software systems with self-* features has been shown to be considerably tougher than traditional, relatively more static and predictable, non-self-* based software systems. A software system with built-in self-reflective properties is said to be self-aware and shows its true picture once subjected to performance. On the other hand, a self-adaptive software system alters its behavior by reflecting on itself [1].

The concept of self-* computing involves different aspects which are subjects of study in research areas of computer science and engineering. Currently, there are technologies that have deployed one or several desirable features of self* computing, such as ability to learn from itself and environment, plan, recommends, or renders explanation. Technologies that exhibit such characteristics can be said to be product of research efforts in areas of artificial intelligence, machine learning, autonomic or cognitive computing. Many of these research disciplines are closely intertwined with research in self-* computing, and they stand to be the current or emerging research topics for todays and future technologies that will be deployed to address emerging human challenges [2]. 
Due to the increasing presence or need of software to drive new or emerging technologies, design of such software poses several challenges. One such challenge that needs surmounted is the ability of the software to manage uncertainty with minimum or without human intervention. Some of the uncertainties may arise during deployment or operation of the technologies, and may include dynamics of resource allocation, changing or evolving operating conditions. Although, designing software systems that drive these new technologies can be highly challenging, it can also be time consuming since complete knowledge about the uncertainties are not handy at development time. Thus, lack of full knowledge of the uncertainties can lead to failure or undesired behavior of the software system (e.g. privacy and security could be breached, performance could degrade, and functionality could be compromised), thereby making the software system unreliable [3], [4].

Notwithstanding above challenges, successful deployment of some of the self-* computing capabilities has made software an essential and integrated part of new technologies. Modern computing systems or technologies integrate incorporate software and hardware to address ever-evolving human challenges. For example: mail delivering drones, healthcare robotic systems, unmanned vehicles, and smart homes and grids; these technologies exploit Internet of Things (IoT) technology to monitor, control and manage tasks usually carried out by human [5].

\section{RELATED WORKS}

Self-reflection (or computational reflection) has been mentioned to constitute the foundation of a system that is selfadaptive; that is, a self-adaptive system must self-reflect. Comprehension of computational (or self-) reflection properties is thereby crucial in order to intelligently and predictably develop a self-adaptive system. However, there exist several key challenges that should be addressed in order to apply the principles of computational refection. For instance, data-parallel programs must be scheduled on the host hardware architecture if such technology is to deploy computation reflection [1].

According to [6], a self-adaptive system is described as a system with interplay between its runtime models and adaptation activities. To achieve the said interplay, runtime megamodels must be adopted. A megamodels, therefore, is a typical model that captures both the runtime models and adaptation activities of a system. To ease the development of adaptation engines of such system, [6] presented an executable modeling language by using a model-driven engineering approach.

Similarly, [7] carried out a case study involving the implementation of self-adaptive software architecture using reflective component model (Fractal) and dynamic Aspect Oriented Programming (AOP) approaches in an industrial Web-based system. Evaluation of both approaches showed they were effective for structural architecture adaptations. However, the approaches were less flexible and not supportive of behavioral adaptation.

A study on the development of novel methods, techniques and tools for engineering self-aware IT systems and services was conducted in [8]. The approach is based on the use of online quality of service models integrated into the system components and capturing all service aspects relevant to managing application quality of service and resource efficiency during operation.

[9] Provided solution to the deliberation scheduling problem for aircrafts using Self-Adaptive Cooperative Intelligent Real-Time Control Architecture (SA-CIRCA). SA-CIRCA is a domain-independent architecture for intelligent and self-adaptive autonomous control systems. The architecture is implementable in hard real-time, mission-critical applications.

Also, [10] presented reference architecture and an approach to the development and adaptation of software systems at runtime. The researchers-initiated Software Engineering for Self-adaptive Software which incorporates adaptations with no interruption in the execution. Four modules were considered (development, action plan, adaptation rule and infrastructure) in the development of the model. Also, three technical considerations were addressed. The first was related to the implementation of the model, which was developed in Java programming language. The second refers to the size and logic organization of the model; while the third addressed entity adaptation. This architecture may be 
adequately used in the area of software automation, but will not be applicable in larger environments of development and execution.

A model and architecture for a system that is meant to manage data streams of real-time human resource applications is discussed in [11] and [12]. This real-time Human Resources Information System (HRIS) is responsible for the collection, recording, storage, analyzing and retrieval of data concerning an organization's human resources. The model described in the referenced papers is made up of six modules. Experimental result as presented in [12], proves that the model provides a more appropriate way of dynamic human resource data management for an organization confined to a branch, but not robust enough to handle a larger organization.

[13] presented a comparative study with other models and identifies their benefits and problems with an application in two software development projects. The author's presentation was based on areas and expected results in the Reference Model for Brazilian Software Process Improvement. In their research, a software factory in a public university was used as a case study. The proposed process model has the capability to plan human resources, review the business needs, hire and relocate members. However, the model is not self-adaptive but typically used for human resource management.

[14] and [15] discussed the implementation of techniques for self-adaptive systems. The approach explained in [14] shows an exciting structure based on Fractal platform to separate the adaptation concern from business code. [16] revealed an approach for managing and administering the complexity of dynamically adaptive systems. The approach conjoins aspect-oriented and model-driven methods to allow adaptation validation at runtime.

In another application of self-adapting concept, [17] developed an efficient self-adapting algorithm for colour-image encryption. The self-adapting algorithm was based on chaos and interactions between multiple red, green and blue layers in an image. To achieve the algorithm, two chaos systems and the interactions between the colour layers was exploited to strengthen the cryptosystem, enhance the confusion and diffusion performance of the encryption system. Experimental results show that the algorithm enhanced the encoding efficiency, improve security of cipher-text, among others.

\subsection{Self-Reflective System}

A computationally reflective system [18], [19], [20] must not only be "self-aware", but must also be "self-adaptive". Self-aware means the ability of a system to collect and organize information about its own behavior and environment; while self-adaptive is for a system to reason about its behavior and make adjustments that change it [20]. Accordingly, a computational reflective system is expected to have ability to collect information about its own structure and behavior, reason about its current state and capabilities in order to enable mid-stream decision on how to modify, retain and analyze its behavior over extended time periods; it should be able to adjust and optimize its decision processes towards targeted success criteria, as well as change the success criteria in response to detected environmental changes (such as newly introduced purposes or goals) [20], [21].

In the literature, computational reflective systems have been identified to exhibit or implement different types of reflections. Two of possible distinct reflection variations identified are: structural and behavioral. Thus,

- $\quad$ Structural reflection is concerned with the reification of structural program aspects [1], [22];

- $\quad$ Behavioral reflection is concerned with the reification of computations and their behavior [1], [19].

In practice, structural reflection enables access to an application's static structure, such as classes, meta-classes, attributes, and method definitions, while behavioral reflection gives access to the dynamic structures, such as objects' state, messages, and call stack.

Also, [1] and [23] identifies two types of reflection at the architectural level that are closely related: topological and strategic.

(a) Topological reflection is capability of a system to perform computations about its own configuration. For example, the addition and removal of architectural elements. 
(b) Strategic reflection deals with system coordination. Example, observations on state of an object may trigger change to some coordination policy.

At architectural level, these latter types of reflection (i.e. topological and strategic reflections) realize structural and behavioral reflections respectively.

\subsection{Self Adaptive Systems}

As mentioned above, self-adaptive system make-sense about its runtime behavior and undertake necessary adjustment that changes it. However, the development of such system is somewhat complex because it has to constantly change structural and/or behavioral features at runtime in other to meet users or execution environment needs [10]. In view of this, a number of software engineering models, techniques, methods, and approaches have been designed and/or deployed. For instance, [24] developed a software engineering process for self-adaptive system; the process is composed of a modeling approach and framework for evaluation, which uses the Software and Systems Process Engineering Meta-Model Specification (SPEM) that was developed by the Object Management Group (OMG) (see [25]).

Generally, self-adaptive system analyses its own performance and changes the performance when the analysis reveals that it is not doing justice to what the software is intended to do, or when better functionality or performance is possible. This simply means that the software has multiple ways of achieving its purpose, and has enough ability of its design and construction to make efficient changes at runtime. Such software should make room for functionality for analyzing its action and performance, as well as the competence to re-examine and re-configure its operations in order to upgrade its operation. Self-adaptive system should also consist of a set of components for each major function, along with narrations of the components, so that components of systems can be selected and scheduled at runtime, in response to the evaluators. [26].

Also, [27] (as referenced in [10]) presented reference architecture for self-adaptive system; the suggested architecture is made-up of a core for adaptation (represented by dotted lines) and four complementary modules (the development, action plan, adaptation rule, and infrastructure modules) as shown in Figure 1 below. The adaptation module (see Figure 1) assists the adaptation activity of the software entities. For this to be achieved, it must be able to control: dynamic behavior, individual reasons, and execution state in relation to the execution environment.

Furthermore, [10], [26], and [6] adopted MAPE-K (Monitor, Analyze, Plan, Execute, and knowledge) control loop as a solution to manage the software adaptation at runtime. The researchers went one step further to summarize the control loop as shown in Figure 2, thus:

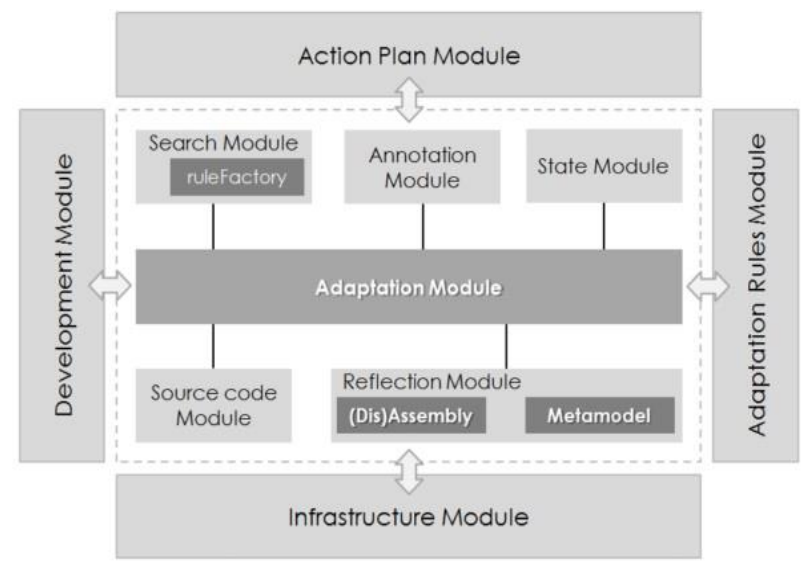

Figure. 1: Reference Architecture for Self-Adaptive System [27]

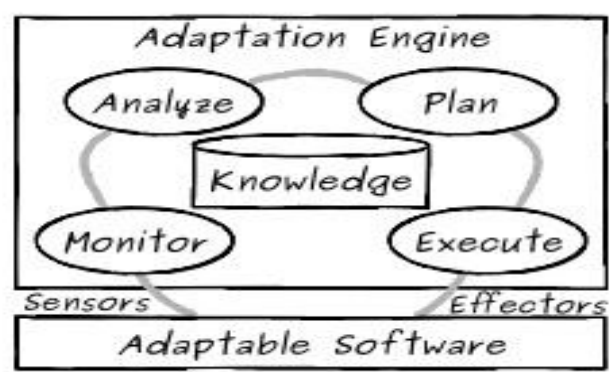

Figure. 2: MAPE-K Control Loop [6] 
(i) The Monitor process has mechanisms that gather data from sensors and convert them to behavioral patterns and symptoms;

(ii) The Plan process creates what will be adapted and how to apply the changes in the software entity in order to get the best outcome;

(iii) The Execute process provides the mechanisms to execute the established action plan;

(iv) The Sensors and Effectors generate data that reflect the states of the system;

(v) The Leave Management involves employees' leaves details which may include holiday or vacation leave, sick leave, maternity leave, etc.

(vi) The Resignation module has the details of the ex-employees who have worked for the organization. Such information includes: department, position, etc

\subsection{Human Resource Management System (HRMS)}

This section is basically concerned with managing the administration of human resource department in an organization. According to [11] and [12], HRMS refers to the systems and processes at the intersection between human resource management (HRM) and information technology. This system is also used to analyze and retrieve information concerning an organization's human resources. The researchers went one step further to develop a model for HRM, and this model consists of six modules:

(i) Employee Details: This module is used to maintain and update the employment details of employees, such as: personal information, contact information and employee status;

(ii) The Payroll module contains the information about the employees' salaries, wages, allowances, etc.;

(iii) The Training module involves the information about employees' schedule, i.e. training for a particular employee conducted by the organization;

(iv) The Performance module has the knowledge of information about the employee's current position in the organization;

(v) The Leave Management involves employees' leaves details. Such as: sick leave, vacation, and holiday; and

(vi) The Resignation module has the details of the ex-employees who have worked for the organization. Such information includes: department, position, etc.

\section{CASE STUDY}

We first discuss briefly the case study used in this research. In this work, for the purpose of implementation and evaluation of our software model, the education domain is chosen with specific consideration of human resource and management. In this scenario, we consider the operational modalities in which teaching and learning process is based on subject teachers, teaching staff and students' populations can increase or decrease at any time due to known and unknown variables, and the population of the students $(\mathbf{x})$ determines the expected number of teachers that a particular school should possess. A secondary school could be understaffed, overstaffed or balanced as the case may be when comparing the actual number of available teachers $\left(\mathbf{y}_{\mathbf{a}}\right)$ to the expected number of teachers $\left(\mathbf{y}_{\mathbf{e}}\right)$.

\subsection{The Software Model: Mathematical Model}

Based on the approach of [28], we developed a software embeddable mathematical model that can be implemented in HRMS for above described scenario. In the model, it is assumed that $\mathbf{x}$ is students population in a school, $\mathbf{i}$ is a specific subject offered by a student (e.g. Math, English, etc.) and $\mathbf{x}_{\mathbf{i}}$ number of students offering a specific subject $\mathbf{i}$.

$$
x_{f} \text { and } x_{m} \subseteq x
$$

where $x_{f}$ and $x_{m}$ are populations of female and male students respectively in a specific school. 
Equation (1) with respect to specific subject will be,

$$
x=\sum_{i=1}^{n} x_{i}=\sum_{i=1}^{n}\left(x_{m_{i}}+x_{f_{i}}\right)
$$

where $m_{i}$ and $f_{i}$ are male and female populations per ith subject.

Also, let $k$ represents the ratio of teacher to students (also known as "Standard"); $y_{a}$ represents the actual number of teachers in a school; and $y_{e}$ represents the expected number of teachers in a school.

Thus, for an entire school, the human resource requirement can be modeled as,

$$
\text { if } y_{a} \neq y_{e}
$$

With regards to equation (3), $\mathcal{Y}_{a}$ for individual subject can be represented thus,

$$
\mathcal{Y}_{a}=\sum_{i=1}^{n} \mathcal{y}_{a_{i}}
$$

such that $a_{i}$ is the actual number of staff per ith subject;

While $\mathcal{Y}_{e}$ will be expressed as,

$$
\mathcal{Y}_{e}=\mathcal{K} X
$$

Thus, equation (4) for individual subject will be:

$$
\mathcal{Y}_{e}=\sum_{i=1}^{n} \mathcal{y}_{e_{i}}=\mathcal{K}\left(\sum_{i=1}^{n} x_{i}\right)
$$

where $e_{i}$ is the expected number of staff per ith subject.

Then,

$$
\begin{aligned}
& \mathcal{Y}_{a}-\mathcal{K} \mathcal{X} \neq 0 \ldots \ldots \ldots \\
& \mathcal{Y}_{a}-\mathcal{K}\left(\sum_{i=1}^{n} x_{i}\right) \neq 0
\end{aligned}
$$

where $i$ stands for each subject.

Due to equation (5), two conditions can arise thus:

$$
\begin{aligned}
& \mathcal{Y}_{a}-\mathcal{K} \mathcal{X}<0 \\
& \text { (6); implies understaffing } \\
& \mathcal{Y}_{a}-\mathcal{K} \mathcal{X}>0 \\
& \text { (7); implies overstaffing. }
\end{aligned}
$$

Note that equations (3) and (4) apply to entire student population per school, while their derivatives 3.1 and 4.1 can be adopted for individual subject per school as shown above. 


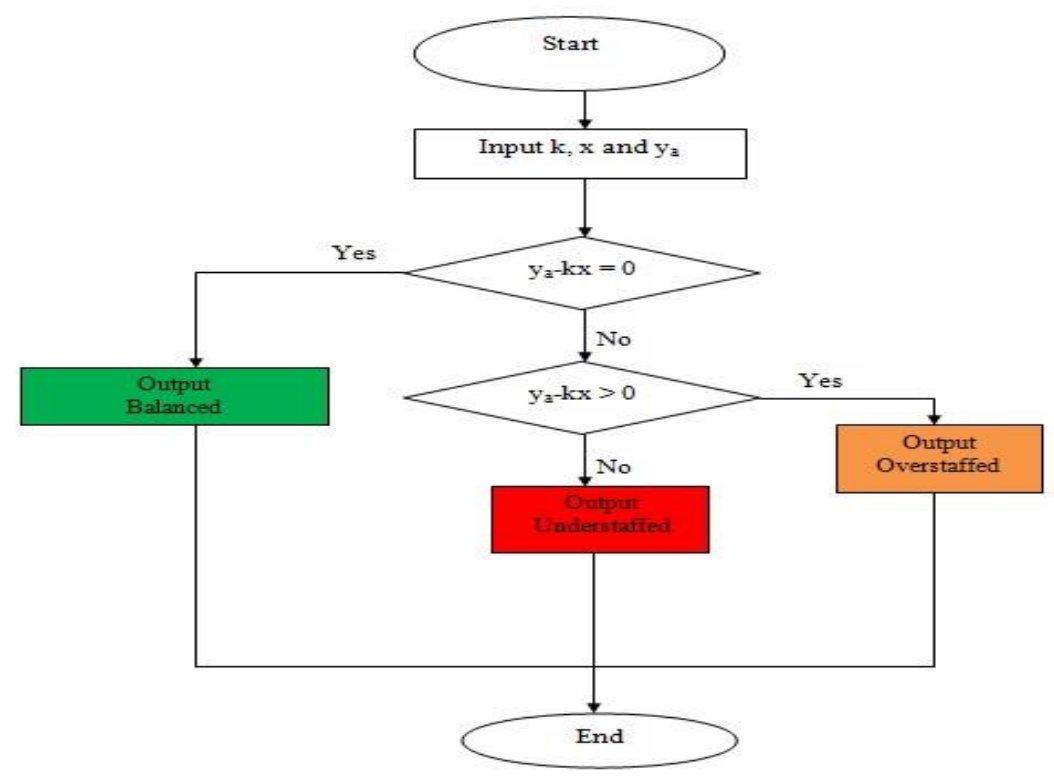

Figure.3: Flow Chart of the Mathematical Model

\subsection{The Software model: Implementation's Visual Models}

In this research, equation (4) and its derivative (4.1) dynamically subject its host software system into self-reflective through continuous monitoring of the value of $\mathbf{x}$ and computation of $\mathbf{y}$ at school and subject levels respectively. This displays the true picture of the system. In this phase, monitoring of relevant state is achieved. Equation (5) and its derivative (5.1) make the system to be a self-adaptive system in that re-distribution of human resource or determination of recruitment need can be undertaken. The built-in knowledge of equations (5) and (5.1) assist in monitoring, analyzing, planning and executing the system modules. Flowing from equations (4) and (5), and their derivatives, equations (6) and (7) can be are generated.

Since data is involved in this research, an open source relational database management system that is based on Structure Query Language is used to develop the database for the model for a human resource management system (HRMS). Thus, the database Entity-relationship model and the architectural model of the HRMS that implements the above mathematical model are presented below.

\subsubsection{The ER-Model.}

An Entity-relationship model (ER-Model) describes the framework of a database with the help of a diagram called the Entity-relationship diagram (ER-Diagram). This diagram is a visual representation of data that describes how data is related to one another. In this work, Figure 4 represents the ER-model of the self-reflect self-adaptive HRMS under consideration.

The database used in this ER-model includes: Admin, Schools, Teachers and Students Population. In the ER-model, the following roles are established:

i. $\quad$ The Admin manages the system;

ii. Teachers teach students;

iii. Teachers teach in a school; and

iv. Students enroll in a school. 


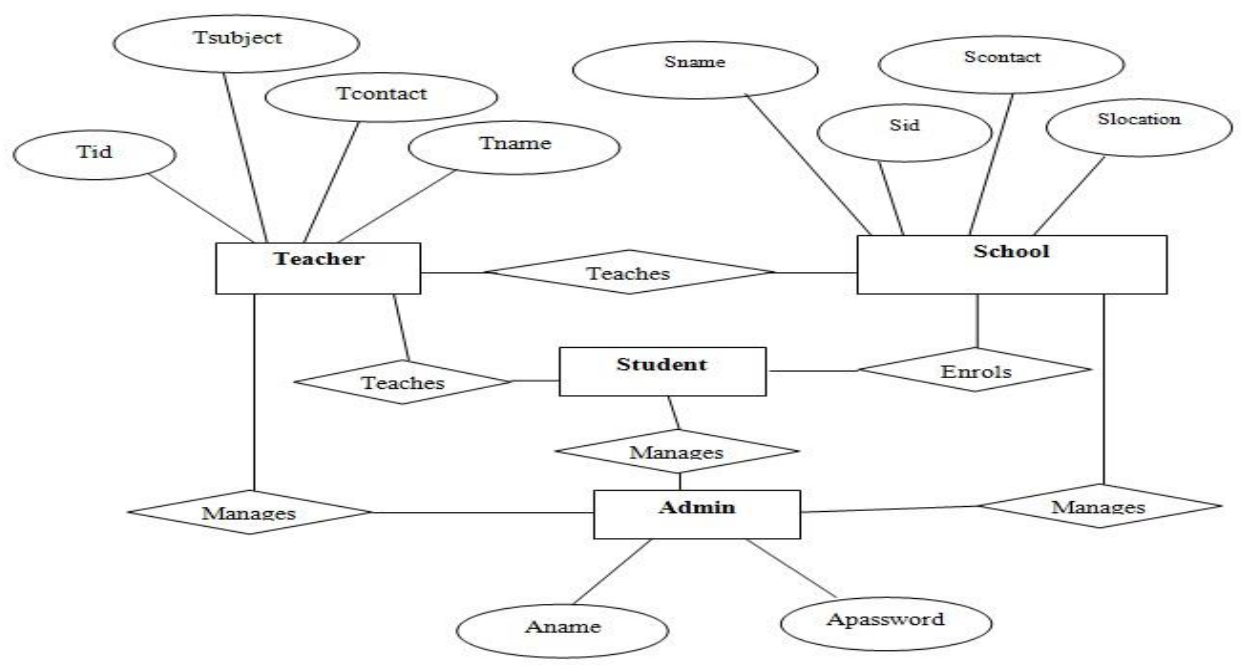

Figure. 4: ER-Model for Self-reflect self-adaptive HRMS

\subsubsection{Architectural Model Description.}

The developed self-reflect self-adaptive HRMS model is a web based model that is completely software based. This model is developed based on the approach of [6]. Apparently, the model consists of five modules and each module has other modules. MySQL, PHP, JavaScript and jQuery, Cascading Style Sheet (CSS), HTML 5 and Bootstrap are used to develop this model for implementation and evaluation.

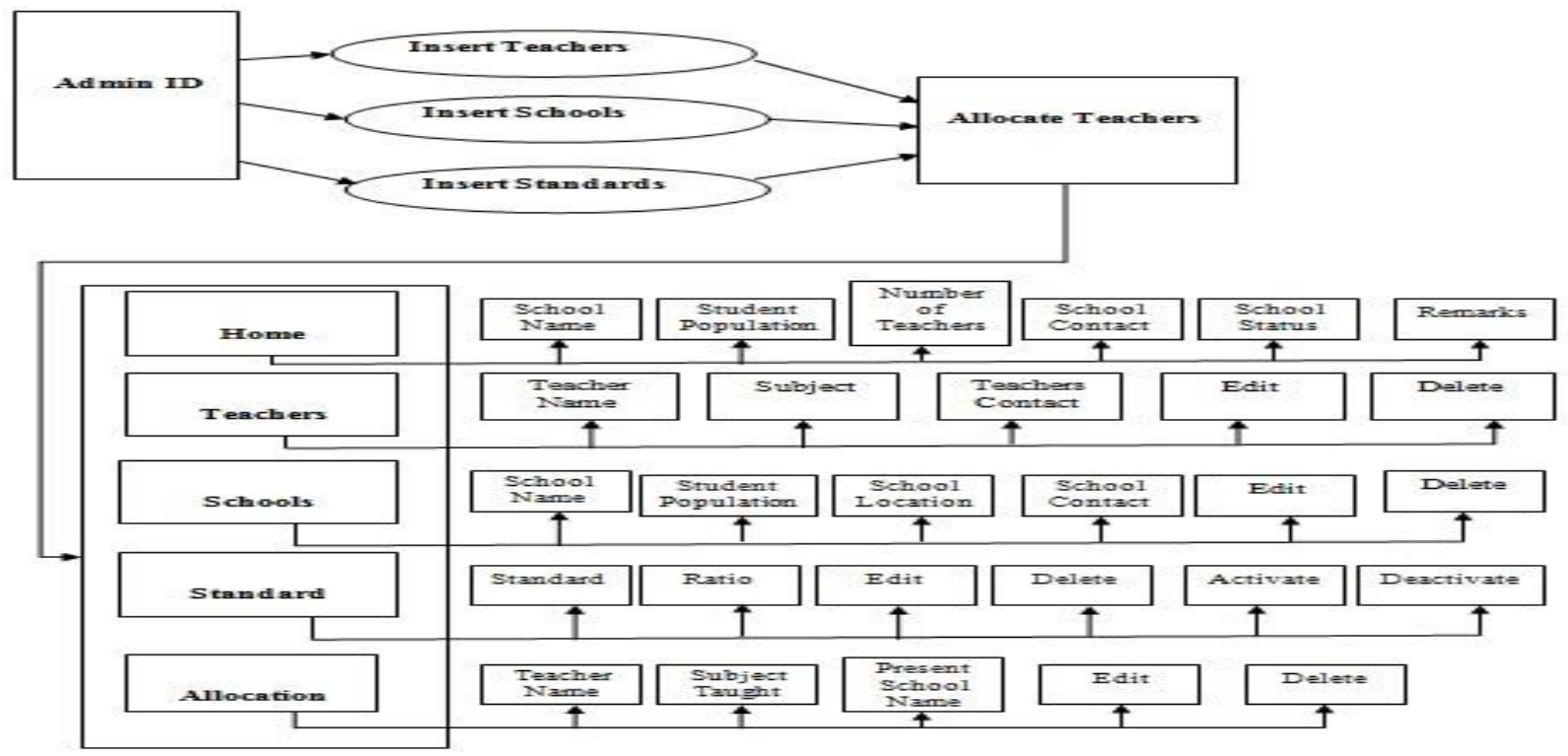

Figure. 5: Visual Architectural Model of Self-reflective self-adaptive HRMS 
The above visual model (i,e. figure 5) has explicit functions; The Allocation, Standard, School, Teachers and Home modules. Hence, the self-reflective self-adaptive can be said to be a meta-model.

Allocation Module: The allocation module consists of the name of teachers and subject taught. This module contains the name of the school at which each subject teacher is allocated to. This is the part of the model in which transfers of subject teachers from one school to another can be initiated. Thus, via the "Allocate Teachers" icon, a form used for posting teachers to school(s) must be filled. The information on this form is displayed in the allocation module.

Standard Module: In this standard module, the "Standard Detail Form" located in the "insert Standard" icon has to be filled. This is where the ratio of number students to teacher is set and the information is displayed in the standard module

Schools Module: This module contains the information about the schools such as the name of each school, the total population of all the students of each school, the location of each school and the school contact. The "insert School" icon displays the "School Detail Form" and the information filled in this form is displayed in the schools module.

Teachers Module: The teachers module contains the information about all the teachers and subject taught. The phone contact of each teacher is also included in this module. This module handles when a teacher retires of leave the system. The "insert Teacher" icon contains "Teacher Detail Form" and this form consists of teacher name, subject taught, email and contact number.

Home Module: The home page of the model shows the real time activity and clearer picture of human resource of each school. The module shows if the teachers of each school short are understaffed, adequate or overstaffed as the case may be.

\section{RESULTS AND DISCUSSIONS}

Figure 6a displays the login page of the implemented model. This is the first interface that was developed. This interface is developed using PHP programming language. This interface is developed for security reasons in order to protect the model. The administrator must log in to this page before the entire self-reflective self-adaptive HRM system can be accessed.
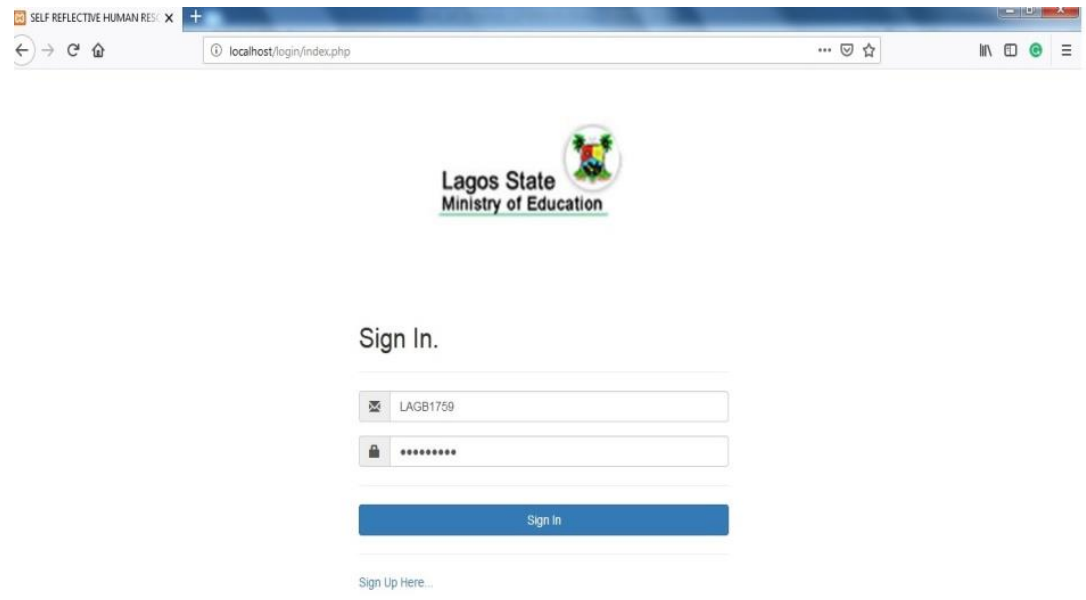

Figure. 6a: Login Screen

Figure $6 \mathrm{~b}$ below shows the output, after inputting the information of the names of teachers, subject taught and total student populations in some junior secondary schools in Lagos State. The system model that leads to the expected 
scenarios have been considered during the development of this model. This Figure $6 \mathrm{~b}$ displays the overview of the model.

Also in this Figure $6 \mathrm{~b}$, the red and orange status describes the schools that are understaffed or overstaffed. The remarks representation gives the number of required teachers or the number of teachers needed to be transferred in or out as the case may be. The status will show green when there is adequate number of allocated teachers to compliment the students' population.

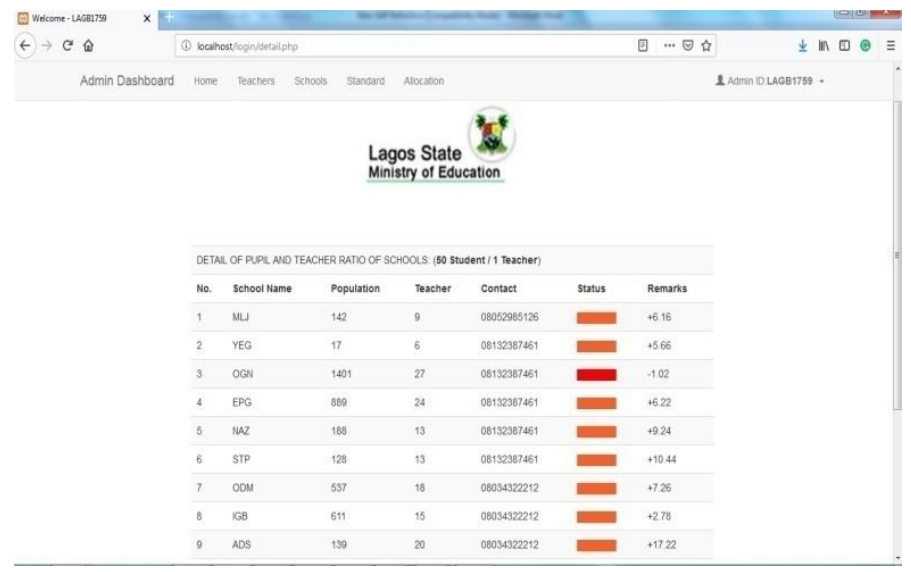

Figure. 6b: Home Screen

The School Data Page in Figure 6c below displays the list of subjects allocated to teachers in a particular school. Since reflection is computations about computations, which means, a computational system performs computation about its own computation. A computational system is expected to give a self-representation of itself [1]. This Figure shows that the system is a reflective system.

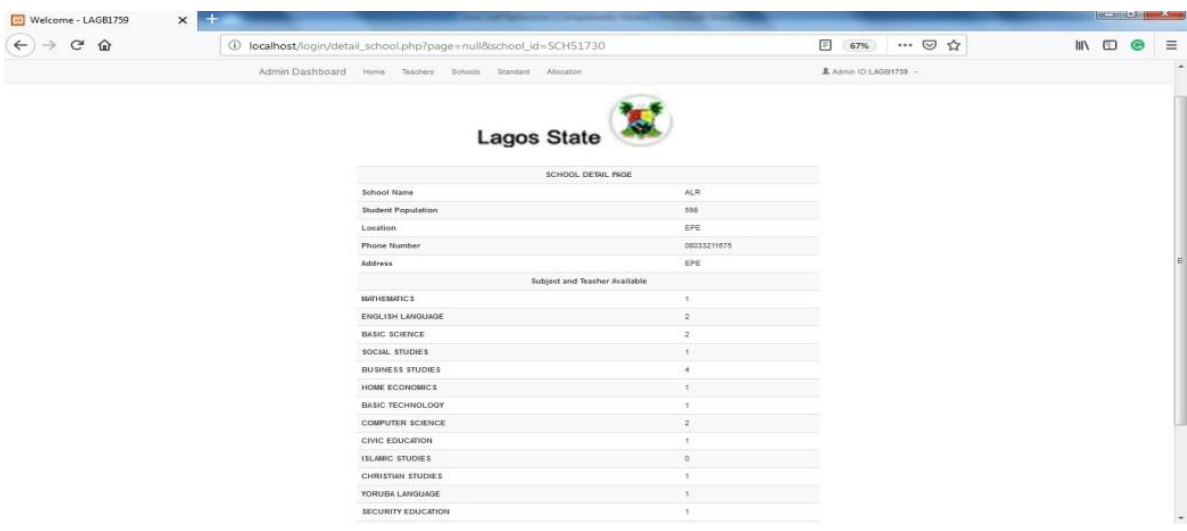

Figure. 6c: School Data Page

\subsection{MATLAB Application for the Mathematical Model}

In addition to the development of the above software, coding of the system model using the MATLAB application was further undertaken. MATLAB R2014a serial update 2 was used for the development of this model (see Figure $7 a)$. 
Figure $7 \mathrm{~b}$ shows the graphical representation of the model. The part that shows $\mathcal{Y}_{a}-\mathcal{K} \mathcal{X}>0$ indicates that region is a region for overstaffing. This simply means that more teachers need to be transferred to other schools or more students needed to be admitted in order to complement the available number of teachers.

The part that signifies $\mathcal{Y}_{a}-\mathcal{K} X<0$ indicates the region of understaffing. This implies that the admitted number of students is more than the available number of teachers in a school. Hence, more teachers are needed in the school.

The linear part of the graph, which is the green line, indicates that there is adequate number of teachers that compliments the admitted number of students. This is an excellent situation and all schools needs to work towards the green.

The farther the schools are drifting away from the green line in any of the cases, the more the problems arise. However, the closer the schools are moving towards the green line, the better for the school.

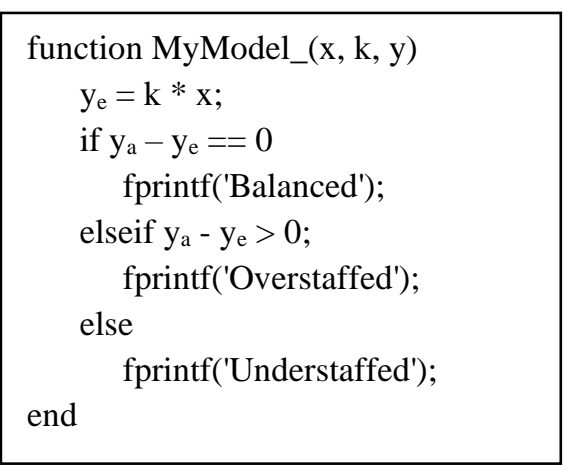

Figure.7a: Implementation of the Mathematical Model

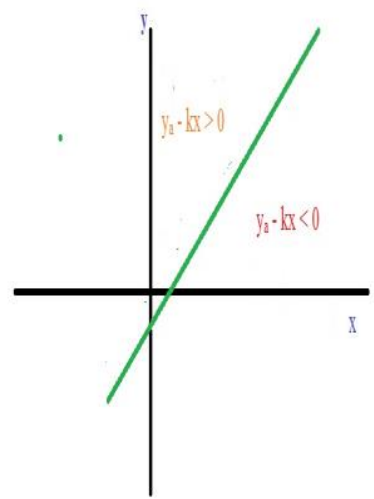

Figure. 7b: Mathematical Model Graph

As earlier discussed, using MATLAB application, Figure 7c represents the graphical outlook of results captured in Figure $6 \mathrm{~b}$ above. Thus, Figure $7 \mathrm{c}$ shows that the region $\mathcal{Y}_{a}-\mathcal{K} \mathcal{X}>0$ indicates overstaffing of teachers. Hence, more students are needed in those schools or some teachers need to be transferred to schools that need more resources. On the other hand, the region $\mathcal{Y}_{a}-\mathcal{K} \mathcal{X}<0$ shows that two schools are understaffed. It is recommended that more teachers are needed in those schools. In an excellent situation, the schools are expected to be on the green line.

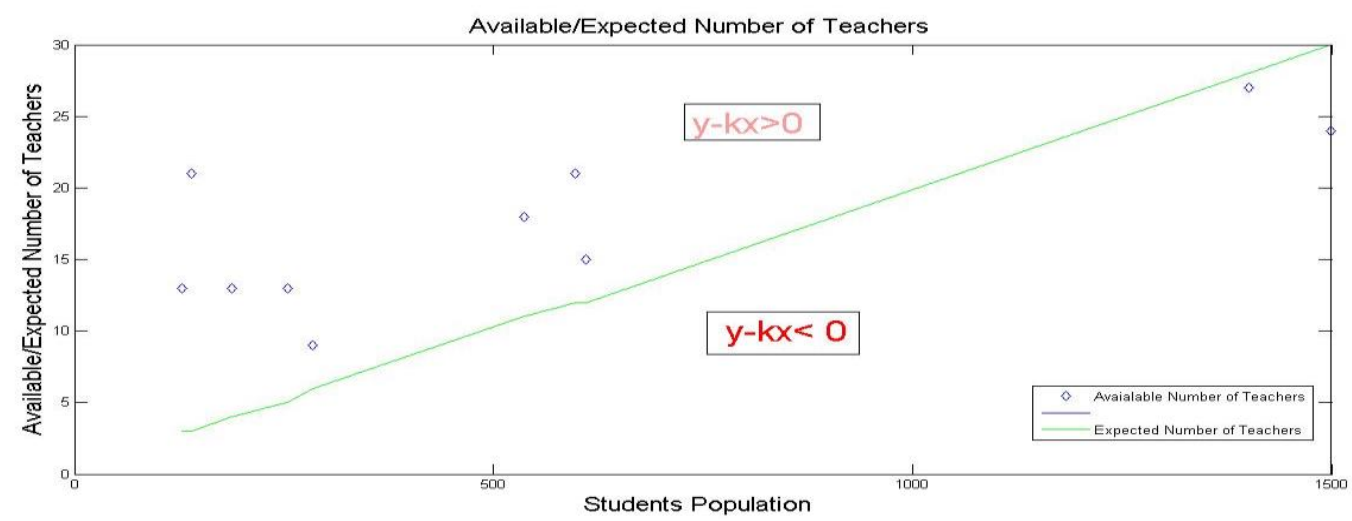

Figure. 7c: Available/Expected Number of Teachers and Students Population 


\section{CONCLUSION}

Existing literatures attest to significant efforts made to design and develop self-reflective and self- adaptive systems. However, most of these systems address "adaptivity" with varying interests including performance and fault management. One of the critical aspects that need more focus is reflective and adaptive systems for human resource management.

In this report, we developed a software model which guides us in the development of visual models. The visual models informed the web-based system developed. Obviously, the model is a software based system that provides the realtime activity of the human resources of a particular school. The data collected is used to analyze the performance of the model. The model is very robust to accommodate more human resource when there is an expansion in the future.

Although, the self reflective and self adaptive systems developed by the previous researchers involve little human intervention and the case studies used are cameras, robots, e.t.c. In our research, little human intervention will still be required because the model deals with human resource.

Limitations and Future Works: The data set used in this research was collected from some schools in Lagos State, although we could not get the comprehensive data of all Secondary Schools in Lagos State. However, provision has already been made that will accommodate as many schools as possible in our model.

The research results presented in this work opens quite a number of good opportunities for future work. An astounding aspect for future research is to enhance the model with high level abstraction and plunge it into more complex industrial reflective and adaptive systems. This will allow to further test the appropriateness of both the behavior and property specification patterns, and their applicability to larger-scale applications.

\section{REFERENCES}

[1] J. Andersson, R. De Lemos, R. de Lemos, S. Malek, and D. Weyns, "Reflecting on Self-adaptive Software Systems.” 2009 ICSE Workshop on Software Engineering for Adaptive and Self-Managing Systems, Vancouver, Canada, May 18-19, 2009, 38-47. doi:10.1109/seams.2009.5069072.

[2] J. Camara et al., "Self-aware computing systems," Self-Aware Computing Systems: Related Concepts and Research Areas, 2017, 17-49. https://doi.org/10.1007/978-3-319-47474-8_2.

[3] M. U. Iftikhar and D. Weyns, "ActivFORMS: active formal models for self-adaptation," in In Proceedings of the 9th International Symposium on Software Engineering for Adaptive and Self-Managing Systems (SEAMS'14), June 2014, 125-134. https://doi.org/10.1145/2593929.2593944.

[4] D. G. de la Iglesia, "A Formal Approach for Designing Distributed Self-Adaptive Systems." Doctoral Dissertation, Department of Media Technology, Linnaeus University, Sweden. Linnaeus University Press, 2014.

[5] U. I. Muhammad, “A Model-Based Approach to Engineer Self-Adaptive Systems with Guarantees.” Doctotal Thesis, Linnaeus University, Sweden, 2017.

[6] T. Vogel and H. Giese, "Model-Driven Engineering of Self-Adaptive Software with EUREMA." ACM Transactions on Autonomous and Adaptive Systems (TAAS), 8(4), 2014, 1-33. doi.org/10.1145/2555612

[7] Y. Wu, Y. Wu, X. Peng, and W. Zhao, "Implementing self-adaptive software architecture by reflective component model and dynamic AOP: A case study," Proc. - Int. Conf. Qual. Softw., no. July, pp. 288-293, 2010 .

[8] S. Kounev, "Self-Aware Software and Systems Engineering: A Vision and Research Roadmap." In 
Proceedings of Software Engineering (SE2011), Nachwuchswissenschaftler-Symposium, 2011.

[9] R. P. Goldman, D. J. Musliner, and K. D. Krebsbach, "Managing Online Self-Adaptation in Real-Time Environments." In: Laddaga R., Shrobe H., Robertson P. (eds) Self-Adaptive Software: Applications, 2003. Lecture Notes in Computer Science, vol 2614. Springer, Berlin, Heidelberg, 2003, 6-23. https://doi.org/10.1007/3-540-36554-0_2.

[10] F. J. Affonso and E. Y. Nakagawa, "Self-adaptive software : development approach and automatic process for adaptation at runtime." Revista Brasileira de Computacao Aplicada, 7(1), 2015, 68-84. doi:10.5335/rbca.2015.4224.

[11] A. S. S. Navaz, A. S. S. Fiaz, C. Prabhadevi, V. Sangeetha, and S. Gopalakrishnan, "Human resources management systems," IOSR J. Comput. Eng., vol. 8, no. 4, pp. 62-71, 2013.

[12] W. Chen, “A Framework for Human Resource Information Systems Based on Data Streams,” Int. J. Hybrid Inf. Technol., 7(3), 177-186, 2014. doi:10.14257/ijhit.2014.7.3.18.

[13] F. E. A. Horita, J. D. Brancher, and R. M. De Barros, “A Process Model for Human Resources Management focused on increasing the Quality of Software Development." 24th International Conference of Software Engineering and Knowledge Engineering (SEKE), 2012.

[14] P.-C. David and T. Ledoux, "An Aspect-Oriented Approach for Developing Self-Adaptive Fractal Components." In: Löwe W., Südholt M. (eds) Software Composition. SC 2006. Lecture Notes in Computer Science, vol 4089. Springer, Berlin, Heidelberg, 2006, 82-97. https://doi.org/10.1007/11821946_6.

[15] R. Wolfmger, S. Reiter, D. Dhungana, P. Grünbacher, and H. Prähofer, "Supporting Runtime System Adaptation Through Product Line Engineering and Plug-in Techniques," in Proceedings - 7th International Conference on Composition-Based Software Systems, ICCBSS 2008), Madrid, 2008, 21-30. doi:10.1109/ICCBSS.2008.30.

[16] B. Morin, O. Barais, G. Nain, and J. M. Jézéquel, “Taming Dynamically Adaptive Systems Using Models and Aspects," in Proceedings - International Conference on Software Engineering, 2009, 122-132.

[17] Y. Luo, R. Zhou, J. Liu, S. Qiu and Y. Cao, “ An efficient and self-adapting colour-image encryption algorithm based on chao and interactions among multiple layers." Multimedia Tools and Applications, 77, 2018, 26191-26217. https://doi.org/10.1007/s11042-018-5844-5.

[18] P. Maes, "Computational reflection". The Knowledge Engineering Review, 3(01), 1988, 1-19. doi:10.1017/s0269888900004355

[19] P. Maes, "Concepts and experiments in computational reflection," ACM SIGPLAN Notices, 22(12),1987, 147-155. doi:10.1145/38807.38821.

[20] C. Landauer and K. L. Bellman, "Reflective Systems Need Models at Run Time." In proceedings of 11th International Workshop on Models@run.time, 2016.

[21] K. L. Bellman, C. Landauer, and P. R. Nelson, "Managing variable and cooperative time behavior," in 2010 $13^{\text {th }}$ IEEE International Symposium on Object/Component/Service-Oriented Real-Time Distributed Computing Workshops, Carmona, Seville,May 4-7, 2010, 49-62. doi: 10.1109/ISORCW.2010.12.

[22] J. Ferber, "Computational Reflection in Class Based Object-Oriented Languages." ACM SIGPLAN Notices, 
24(10), 1989, 317-326. doi:10.1145/74878.74910.

[23] W. Cazzola, A. Savigni, A. Sosio, and F. Tisato, "Rule-based strategic reflection: observing and modifying behaviour at the architectural level." 14th IEEE International Conference on Automated Software Engineering, Cocoa Beach, Florida, USA, October 12-15, 1999, 263-266. doi:10.1109/ase.1999.802308.

[24] J. Andersson et al., "Software Engineering Processes for Self-Adaptive Systems.” In: de Lemos R., Giese H., Müller H.A., Shaw M. (eds) Software Engineering for Self-Adaptive Systems II. Lecture Notes in Computer Science, vol 7475, 2013, 51-75. Springer. Berlin, Heidelberg. doi:10.1007/978-3-642-35813$5 \_3$.

[25] (OMG), O. M. G. Software \& Systems Process Engineering Meta-Model Specification (SPEM). 2008. Online.Version 2.0. Avaliable at: <http://www.omg.org/spec/SPEM/2.0/>.

[26] M. Salehie and L. Tahvildari, "Self-adaptive software: Landscape and research challenges.” ACM transactions on autonomous and adaptive systems (TAAS), 4(2), 2009, 1-42. doi.org/10.1145/1516533.1516538

[27] F. J. Affonso and E. Y. Nakagawa, "A Reference Architecture Based on Reflection for Self-Adaptive Software." In 2013 VII Brazilian Symposium on Software Components, Architectures and Reuse, Brasilia, Brazil, Sept. 29 - Oct. 4, 2013, 129-138. doi:10.1109/SBCARS.2013.24.

[28] G. Behrmann, A. David, and K. G. Larsen, “A Tutorial on UPPAAL.” In: Bernardo, M., Corradini, F. (eds) Formal Methods for the Design of Real-Time Systems: SFM-RT 2004, Lecture Notes in Computer Science, Vol 3185, 2004, 200-236. Springer, Berlin, Heidelberg. doi.org/10.1007/978-3-540-30080-9_7. 